\title{
INDUSTRIAL ACTUATOR DIAGNOSIS USING HIERARCHICAL FUZZY NEURAL NETWORKS
}

\author{
M. J. G. C. Mendes", J. M. F. Calado ${ }^{*}$, J. M. Sousa ${ }^{\dagger}$ and J. M. G. Sá da Costa ${ }^{\dagger}$ \\ "IDMEC/ISEL - Instituto Superior de Engenharia de Lisboa \\ Polytechnic Institute of Lisbon, Departamento de Engenharia Mecânica \\ Mechanical Engineering Studies Centre \\ Rua Conselheiro Emídio Navarro, 1949-014 Lisboa, Portugal \\ Fax: + 351218317057 , e-mail: \{mmendes, jcalado\} @ dem.isel.ipl.pt \\ † Technical University of Lisbon, Instituto Superior Técnico \\ Dept. of Mechanical Engineering, GCAR/IDMEC \\ Av. Rovisco Pais, 1049-001 Lisboa, Portugal \\ Fax: + 35121 8498097, e-mail: \{j.sousa, sadacosta\}@gcar.ist.utl.pt
}

Keywords: Fault diagnosis, fuzzy neural network, single faults, multiple simultaneous faults, incipient faults.

\begin{abstract}
In this paper a hierarchical structure of fuzzy neural networks (FNNs) and how to train it for fault isolation given an appropriate data patterns, are presented. Fault symptoms concerning multiple simultaneous faults are harder to learn than those associated with single faults. Furthermore, the larger the set of faults, the larger the set of fault symptoms will be and, hence, the longer and less certain the training outcome. In order to overcome this problem, the proposed approach has a hierarchical structure of three levels where several FNNs are used. Thus, a large number of patterns are divided into many smaller subsets so that the classification can be carried out more efficiently. One of the advantages of this approach is that multiple faults can be detected in new data even if the network is trained only with data representing single abrupt faults. A continuous binary distillation column having several actuated valves with PID loops has been used as test bed for the proposed approach.
\end{abstract}

\section{Introduction}

A major goal of intelligent control systems is to achieve high performance with increasing reliability, availability and automation of maintenance procedures. In many applications, increased requirements on productivity and performance lead to plants operating near design limits for much of the time. This may often result in system failures, which are typically characterised by critical changes in the inherent dynamics of the process. For these reasons, there is a growing need for online fault detection and diagnosis approaches in order to increase reliability of safety-critical industrial processes.

In dynamical systems, faults may be divided into two main classes: abrupt failures and incipient failures. The incipient failures affect the process behaviour slowly and may take a long time before being detected. Conversely, abrupt failures give rise to jumps in the process parameters, resulting in an appreciable deviation from normal system behaviours.

Fault detection and isolation systems based on conventional design techniques are usually supported by linear process models [9]. For non-linear processes, the traditional approach is to linearize the process model around the process operating point. This approach is effective for many non-linear processes if the operating range is limited and the fault detection and isolation system has been designed to be robust enough to tolerate small perturbations around the operating point. However, for processes with high nonlinearities and a wide operating range, the linearized approach fails to give satisfactory results. One solution is to use a large number of linearized models corresponding to a range of operating points which is not very practical for real time applications [3].

Since artificial neural networks (ANNs) can be trained to have the required mapping between inputs and outputs, they can be used to overcome the difficulties of conventional fault detection techniques to deal with nonlinear behaviors. Establishing an appropriate training set allows the neural network to learn and generalize for operating with future input data. This learning capacity makes ANNs suitable for designing diagnosis systems. The number of publication on this subject demonstrates the potentiality of this new tool $[1,2,6,7,9,11]$

In this approach a hierarchical fuzzy neural system that combines the advantages of both fuzzy reasoning and neural networks will be used. Fuzzy reasoning is capable of handling uncertainty and imprecise information, while an ANN is capable of learning from examples. The adoption of a hierarchical fuzzy neural network (HFNN) approach for fault isolation aims at the development of an architecture that can localize abrupt and incipient single and multiple faults correctly, or at least with a minimum misclassification rate, from only single abrupt fault symptoms, and be easily trained. The developed fault isolation system has been successful applied to a continuous binary distillation column (CBDC). 
The paper is organized as follows. Section 2 provides a description of the hierarchical structure of the fuzzy neural networks used for fault isolation purposes, and explains the design of this FNNs structure. Section 3 describes the CBDC process and the results obtained with the developed fault isolation system for single and multiple faults. Finally, in Section 4 some concluding remarks are given.

\section{Hierarchical Structure of Fuzzy Neural Networks}

In the current approach a hierarchical structure of several fuzzy neural networks have been developed for fault isolation purposes $[1,5,8]$. It is aimed to identify multiple simultaneous faults from only single abrupt fault symptoms. The hierarchical structure has three levels where several FNNs are used, as shown in Figure 1.

The lower level consists of one FNN where all variations $(\Delta e)$ of the measured variables are used as inputs. At the medium level a number of FNNs (structurally identical) that is equal to the number of single fault scenarios considered, are used. Each FNN at the medium level is also fed with all the measurement variables and each one is associated with an output of the FNN at the lower level, corresponding to a particular single fault. The upper level consists of an OR operation of the outputs of the FNNs of the medium level.

The elements of the set used in the OR operation are determined by the outputs of the FNN at the lower level. Thus, if the $i$-th and $j$-th outputs of the FNN at the lower level is taking values close to 1 , then the outputs of the $i$-th and $j$-th FNNs at the medium level form the elements used in the OR operation. However, if only one output of the FNN at the lower level is taking a value close to 1 , then the corresponding FNN in the medium level is used to confirm that this fault is a single fault, or to diagnose multiple faults. Obviously, the multiple faults must include the one corresponding to the output of the FNN at the lower level.

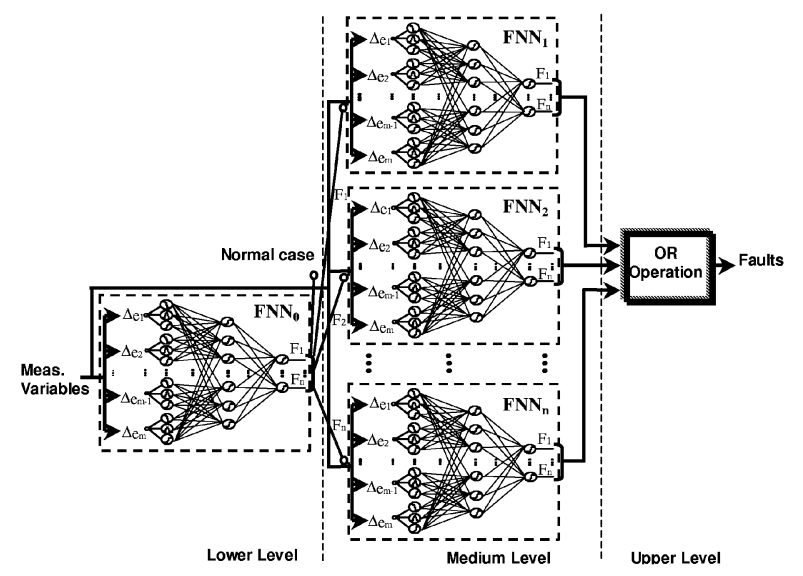

Figure 1: Hierarchical Fuzzy Neural Networks.
In contrast to the conventional multi-layer feedforward neural network, the adopted FNN has an additional fuzzy input layer that maps the increment of each measurement into fuzzy sets. Therefore, the fuzzification layer converts each input into the quantity space, $q_{f}=\{$ decrease, steady, increase $\}$ by association with three types of neurons. The processing elements of the fuzzification layer related to the fuzzy sets decrease and increase use the complement sigmoid function and the sigmoid function, respectively, as their activation functions. On the other hand, the other processing elements of the fuzzification layer related to the fuzzy set steady use the Gaussian function. The hidden and output layers processing elements use the sigmoid function as their activation functions.

Both the lower level and the medium level networks are made up of three layers: a fuzzification layer, a hidden layer, and an output layer. The FNNs are trained using the resilient backpropagation-learning algorithm [10].

The $\mathrm{FNN}_{0}$ training data is obtained from the process single abrupt fault simulation, where abrupt faulty scenarios and the stationary operational conditions are being considered (see Table 1).

On the other hand, the $\mathrm{FNN}_{\mathrm{i}}$ is trained using the data for one single abrupt fault (the fault associated with the corresponding $\mathrm{FNN}_{\mathrm{i}}$ ) and for all possible double abrupt faults that the $\mathrm{FNN}_{\mathrm{i}}$ net will be able to diagnose. This training data is obtained by adding the data for the corresponding single abrupt faults considered, as shown in Table 2 and in the Equation (1).

$$
\Delta e_{1}{ }^{l+2} \ldots \Delta e_{m}{ }^{l+2}=\Delta e_{l}{ }^{l}+\Delta e_{l}{ }^{2} \ldots \Delta e_{m}{ }^{l}+\Delta e_{m}{ }^{2}
$$

\begin{tabular}{|c|c|c|c|}
\hline Vectors & FNN inputs & $\begin{array}{c}\text { FNN } \\
\text { outputs }\end{array}$ & $\begin{array}{c}\text { Fault } \\
\text { diagnosed }\end{array}$ \\
\hline 1 & $\Delta e_{l}^{0} \ldots \Delta e_{m}{ }^{0}$ & $000 \ldots 0$ & Normal \\
\hline 2 & $\Delta e_{1}^{l} \ldots \Delta e_{m}^{l}$ & $100 \ldots 0$ & $\mathrm{~F}_{1}$ \\
\hline . & . & . & . \\
\hline$\cdot$ & . & . & . \\
\hline n-1 & $\Delta e_{1}^{n-1} \ldots \Delta e_{m}^{n-1}$ & $00 \ldots 10$ & $F_{n-1}$ \\
\hline $\mathrm{n}$ & $\Delta e_{1}^{n} \ldots \Delta e_{m}^{n}$ & $000 \ldots 1$ & $F_{n}$ \\
\hline
\end{tabular}

Table 1: Training data for single abrupt faults.

\begin{tabular}{|c|c|c|c|}
\hline Vectors & FNN inputs & $\begin{array}{c}\text { FNN } \\
\text { outputs }\end{array}$ & $\begin{array}{c}\text { Faults } \\
\text { diagnosed }\end{array}$ \\
\hline 1 & $\Delta e_{1}{ }^{I+2} \ldots \Delta e_{m}{ }^{l+2}$ & $110 \ldots 0$ & $\mathrm{~F}_{1} \mathrm{~F}_{2}$ \\
\hline 2 & $\Delta e_{1}^{1+3} \ldots \Delta e_{m}^{1+3}$ & $101 \ldots 0$ & $\mathrm{~F}_{1} \mathrm{~F}_{3}$ \\
\hline . & $\cdot$ & . & . \\
\hline . & . & . & . \\
\hline$\cdot$ & $\cdot$ & $\cdot$ & · \\
\hline $\begin{array}{c}n-1 \\
n\end{array}$ & $\Delta e_{1}^{(n-1)+n} \ldots \Delta e_{m}^{(n-1)+n}$ & $00 \ldots 11$ & $F_{n-1} F_{n}$ \\
\hline
\end{tabular}

Table 2: Training data for double abrupt faults. 


\section{Case Study}

A Continuous Binary Distillation Column, which is described by [4], has been used as a test bed of the fault isolation system proposed in this paper. This process is shown in Figure 2, where a column containing a total of eight theoretical plates plus a reboiler is assumed, with feed entering on plate 5 . Surge drum and reboiler levels are controlled by feedback control loops. The process simulation has been implemented through MATLAB/SIMULINK environment.

The fault diagnosis system is based on a HFNN with the characteristics previously presented. Hence, four measurement variables have been used as input data to all the fuzzy neural networks. These variables are the following: $M_{D}$, hold-up in surge drum; $D$, distillate flow rate; $M_{B}$, hold-up in reboiler; $W$, bottom flow rate. In order to achieve a diagnosis of a fault or faults in the process, an analysis of the output values from the fuzzy neural networks $\mathrm{FNN}_{0}$ is necessary. If the number of nonzero outputs (output $\geq 0,5$ ) in $\mathrm{FNN}_{0}$ is equal to 0 , then it is assumed that no fault occurred in the process under consideration. Otherwise, the result of the HFNN is considered to be the result of an OR operation (upper level) on several $\mathrm{FNN}_{\mathrm{i}}$ outputs in medium level, as previously described.

In the fault isolation system implemented all the FNNs are equal, with a fuzzification layer consisting of 12 processing elements arranged in 4 groups, corresponding to the 4 measurement variables, with each group containing 3 neurones corresponding to the respective fuzzy sets.

The number of neurones in the hidden layer is determined by the complexities of the relationships between the faults and the fault symptoms. During the current study, it was found that 10 hidden processing elements could give good

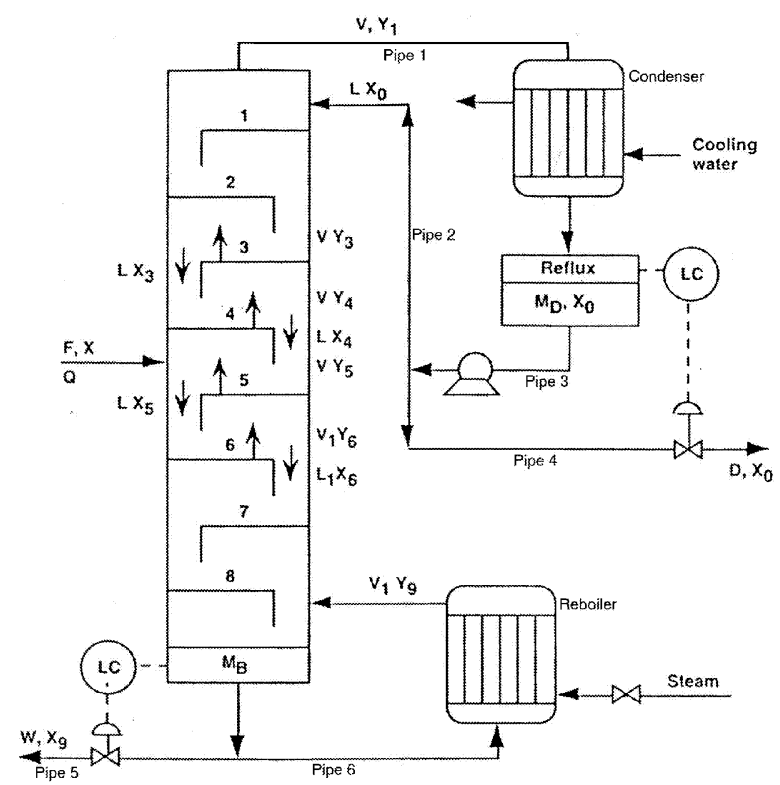

Figure 2: Continuous Binary Distillation Column. performance. Moreover, since 4 single faults have been considered $\left(F_{1}\right.$, valve on pipe 4 blocked fully open; $F_{2}$, valve on pipe 4 blocked fully closed; $F_{3}$, valve on pipe 5 blocked fully open; $F_{4}$, valve on pipe 5 blocked fully closed) the output layer of each network is up of 4 neurones, each one corresponding to a fault. It was also considered all possible double fault scenarios corresponding to an AND operation in the single fault space.

The training data used in this structure was obtained with the aim of covering nine different process-operating points ( $10 \%$ to $90 \%$ of controlled variables). Then, 45 learning patterns are involved in $\mathrm{FNN}_{0}$ training and 27 learning patterns are involved in each $\mathrm{FNN}_{\mathrm{i}}$ training.

The results achieved with the fault diagnosis system are presented in tables (see for example Table 3). These tables have a first column with the faults simulated. A second column, where the classification values $(\mathrm{F} 1=0,9964$, for example) are presented, corresponding to the results achieved with the lower level ( $\mathrm{FNN}_{0}$ network). Furthermore, they also have four columns associated with the four fuzzy neural networks at the medium level and finally, a column with the final diagnosis results of the HFNN structure (achieved after union operation).

The results achieved so far have shown that the HFNN proposed in this paper is a potential tool for fault isolation of single/multiple abrupt (Tables 3, 4 and 5) and incipient faults (Table 6, 8 and 10). During the simulation studies with abrupt faulty scenarios, a very accurate diagnosis has been obtained.

Tables 3, 4 and 5 show the diagnosis results for single and double abrupt faults under different process operating points. From these tables it can be observed that all single and double

\begin{tabular}{|c|c|c|c|c|c|c|}
\hline \multirow{2}{*}{ Faults } & $\begin{array}{c}\text { Lower } \\
\text { Level }\end{array}$ & \multicolumn{4}{|c|}{ Medium Level } & $\begin{array}{c}\text { Upper } \\
\text { Level }\end{array}$ \\
\cline { 2 - 7 } & $\mathrm{FNN}_{0}$ & $\mathrm{FNN}_{1}$ & $\mathrm{FNN}_{2}$ & $\mathrm{FNN}_{3}$ & $\mathrm{FNN}_{4}$ & $\begin{array}{c}\text { OR } \\
\text { Operation }\end{array}$ \\
\hline $\mathrm{F}_{1}$ & $\mathrm{~F}_{1}=0.9964$ & $\mathrm{~F}_{1}$ & - & - & - & $\mathrm{F}_{1}$ \\
\hline $\mathrm{F}_{2}$ & $\mathrm{~F}_{2}=0.9984$ & - & $\mathrm{F}_{2}$ & - & - & $\mathrm{F}_{2}$ \\
\hline $\mathrm{F}_{3}$ & $\mathrm{~F}_{3}=0.9944$ & - & - & $\mathrm{F}_{3}$ & - & $\mathrm{F}_{3}$ \\
\hline $\mathrm{F}_{4}$ & $\mathrm{~F}_{4}=0.9961$ & - & - & - & $\mathrm{F}_{4}$ & $\mathrm{~F}_{4}$ \\
\hline
\end{tabular}

Table 3: Diagnosis results of single abrupt faults $\left(M_{D}=M_{B}=10 \%\right.$ of $M_{D}$ and $M_{B}$ maximum).

\begin{tabular}{|c|c|c|c|c|c|c|}
\hline \multirow{2}{*}{ Faults } & $\begin{array}{c}\text { Lower } \\
\text { Level }\end{array}$ & \multicolumn{4}{|c|}{ Medium Level } & $\begin{array}{c}\text { Upper } \\
\text { Level }\end{array}$ \\
\cline { 2 - 7 } & $\mathrm{FNN}_{0}$ & $\mathrm{FNN}_{1}$ & $\mathrm{FNN}_{2}$ & $\mathrm{FNN}_{3}$ & $\mathrm{FNN}_{4}$ & $\begin{array}{c}\text { OR } \\
\text { Operation }\end{array}$ \\
\hline $\mathrm{F}_{1} \mathrm{~F}_{3}$ & $\begin{array}{c}\mathrm{F}_{1}=0.9163 \\
\mathrm{~F}_{3}=0.8790\end{array}$ & $\mathrm{~F}_{1} \mathrm{~F}_{3}$ & - & $\mathrm{F}_{1} \mathrm{~F}_{3}$ & - & $\mathrm{F}_{1} \mathrm{~F}_{3}$ \\
\hline $\mathrm{F}_{1} \mathrm{~F}_{4}$ & $\begin{array}{c}\mathrm{F}_{1}=0 \\
\mathrm{~F}_{4}=0.9922\end{array}$ & - & - & - & $\mathrm{F}_{1} \mathrm{~F}_{4}$ & $\mathrm{~F}_{1} \mathrm{~F}_{4}$ \\
\hline $\mathrm{F}_{2} \mathrm{~F}_{3}$ & $\begin{array}{c}\mathrm{F}_{2}=0.9930 \\
\mathrm{~F}_{3}=0.6749\end{array}$ & - & $\mathrm{F}_{2} \mathrm{~F}_{3}$ & $\mathrm{~F}_{2} \mathrm{~F}_{3}$ & - & $\mathrm{F}_{2} \mathrm{~F}_{3}$ \\
\hline $\mathrm{F}_{2} \mathrm{~F}_{4}$ & $\begin{array}{c}\mathrm{F}_{2}=0 \\
\mathrm{~F}_{4}=0.9851\end{array}$ & - & - & - & $\mathrm{F}_{2} \mathrm{~F}_{4}$ & $\mathrm{~F}_{2} \mathrm{~F}_{4}$ \\
\hline
\end{tabular}

Table 4: Diagnosis results of double abrupt faults ( $M_{D}=M_{B}=10 \%$ of $M_{D}$ and $M_{B}$ maximum). 


\begin{tabular}{|c|c|c|c|c|c|c|}
\hline \multirow{2}{*}{ Faults } & $\begin{array}{c}\text { Lower } \\
\text { Level }\end{array}$ & \multicolumn{4}{|c|}{ Medium Level } & Upper Level \\
\cline { 2 - 7 } & $F_{N N}$ & $F_{0 N}$ & $F_{1} N_{2}$ & $F_{N N}$ & $F_{1} N_{4}$ & $\begin{array}{c}\text { OR } \\
\text { Operation }\end{array}$ \\
\hline$F_{1} F_{3}$ & $F_{1} F_{3}$ & $F_{1} F_{3}$ & - & $F_{1} F_{3}$ & - & $F_{1} F_{3}$ \\
\hline$F_{1} F_{4}$ & $F_{4}$ & - & - & - & $F_{1} F_{4}$ & $F_{1} F_{4}$ \\
\hline$F_{2} F_{3}$ & $F_{2} F_{3}$ & - & $F_{2} F_{3}$ & $F_{2} F_{3}$ & - & $F_{2} F_{3}$ \\
\hline$F_{2} F_{4}$ & $F_{4}$ & - & - & - & $F_{2} F_{4}$ & $F_{2} F_{4}$ \\
\hline
\end{tabular}

\begin{tabular}{|c|c|c|c|c|c|c|}
\hline \multirow{2}{*}{ Faults } & $\begin{array}{c}\text { Lower } \\
\text { Level }\end{array}$ & \multicolumn{4}{|c|}{ Medium Level } & $\begin{array}{c}\text { Upper } \\
\text { Level }\end{array}$ \\
\cline { 2 - 7 } & $F_{N}$ & $F_{0 N}$ & $F N N_{2}$ & $F_{N N}$ & $F$ & $\begin{array}{c}\text { OR } \\
\text { Operation }\end{array}$ \\
\hline$F_{1}$ & $F_{1}=0.5059$ & $F_{1}$ & - & - & - & $F_{1}$ \\
\hline$F_{2}$ & $F_{2}=0.5005$ & - & $F_{2}$ & - & - & $F_{2}$ \\
\hline$F_{3}$ & $F_{3}=0.5038$ & - & - & $F_{3}$ & - & $F_{3}$ \\
\hline$F_{4}$ & $F_{4}=0.5080$ & - & - & - & $F_{4}$ & $F_{4}$ \\
\hline
\end{tabular}

Table 5: Diagnosis results of double abrupt faults $\left(M_{D}=90 \%\right.$, $M_{B}=10 \%$ of $M_{D}$ and $M_{B}$ maximum).

abrupt faults are diagnosed correctly. It can also be conclude that the behaviour of the diagnosis structure does not change with the different process levels $\left(M_{D}\right.$ and $\left.M_{B}\right)$.

In industrial processes, it is common that the components degradation (Dc) follow an exponential law (for example the valves), giving origin to incipient faults. However, in this work (in the incipient faults simulation) it was considered that the components degradation of the CBDC follow a linear law, because this is the case for which it is more difficult to diagnose the consequent incipient faults (see Figure 3).

The Figure 3 presents the different types of components degradation that gives origin to abrupt and incipient faults. $D_{N}$ represents the normal behaviour or a null degradation in the components, and $\mathrm{D}_{\max }$ represents the maximum degradation in the components of the process. In the CBDC, faults are simulated through the abrupt or incipient change in the process variables that are affected by the faults. Thus, considering for example the $F_{1}$ and $F_{2}$ faults that happen on valve of tube 4 , these are simulated through the abrupt or incipient change in the flow $D$. The HFNN structure that aims the faults isolation in the process will have the capability to deal with incipient faults. Therefore, it is important to achieve the minimum slope to the component degradation linear law for which the fault isolation approach still able to diagnose the correct fault. Thus, in the incipient faults simulation the slope of the linear law corresponding to the component degradation was chosen to be the minimum such that the faults can be isolated. Tables 6 to 11 show the diagnosis results for single and double incipient faults under some

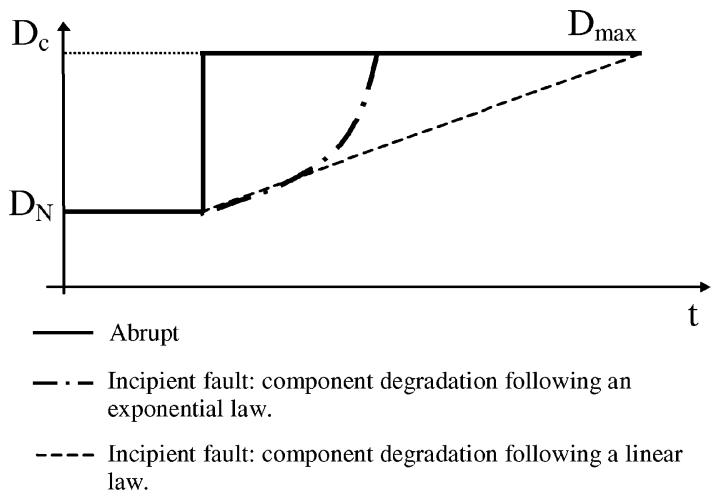

Figure 3: Component degradation laws.

Table 6: Diagnosis results of single incipient faults $\left(M_{D}=M_{B}=10 \%\right.$ of $M_{D}$ and $M_{B}$ maximum $)$

process operating point and it can observed that all incipient faults were diagnosed correctly.

It has also been observed that the neural network's generalisation ability has a great importance in the diagnosis of incipient faults since the training patterns only include single abrupt fault symptoms for a limited number of process operating points.

In order to measure the HFNN structure performance under incipient fault scenarios the following criterion has been used: with the slope of the component degradation linear law taking the minimum value that allows the fault isolation system diagnose the correct fault, the fault isolation time has been measured. All tests have been performed using a PC Pentium III, $450 \mathrm{MHz}$ and fitted with $8 \mathrm{Mb}$ RAM.

Furthermore, it has been observed that the minimum value to the slope of the component degradation linear law, mentioned above, depends on the values taken by the controlled process variables. Thus, two situations have been considered, one with the controlled process variables taking the value $10 \%$ and another taking the value 90\%. From Tables 6 and 8 can be seen that for all four single faults considered the correct diagnosis has been achieved. The $\mathrm{FNN}_{0}$ outputs are taking values close to 0.5 , which is the used value as a decision criterion for the occurrence of a fault. Therefore, the corresponding FNNs in the medium level will be active and confirm the correct fault occurrence as shown in Tables 6 and 8

The results shown in Table 6 have been achieved under incipient fault scenarios, as previously mentioned, with the slope of the component degradation linear law taking the values presented in Table 7. This Table also shows the fault isolation times achieved for the different incipient fault situations. It can be observed that the slow component

\begin{tabular}{|c|c|c|}
\hline Incipient faults & Minimal slope & Diagnosis time $[\mathrm{s}]$ \\
\hline $\mathrm{F}_{1}$ & 91.0 & 0.93 \\
\hline $\mathrm{F}_{2}$ & -9.8 & 8.56 \\
\hline $\mathrm{F}_{3}$ & 37.0 & 2.07 \\
\hline $\mathrm{F}_{4}$ & -4.5 & 17.56 \\
\hline
\end{tabular}

Table 7: Minimum slopes and diagnosis time for single incipient faults $\left(M_{D}=M_{B}=10 \%\right.$ of $M_{D}$ and $M_{B}$ maximum). 


\begin{tabular}{|c|c|c|c|c|c|c|}
\hline \multirow{2}{*}{ Faults } & Lower & \multicolumn{4}{|c|}{ Medium Level } & Upper \\
\hline & $\mathrm{FNN}_{0}$ & $\mathrm{FNN}_{1}$ & $\mathrm{FNN}_{2}$ & $\mathrm{FNN}_{3}$ & $\mathrm{FNN}_{4}$ & $\begin{array}{c}\text { OR } \\
\text { Operation }\end{array}$ \\
\hline$F_{1}$ & $F_{1}=0,5029$ & $\mathrm{~F}_{1}$ & - & - & - & $\mathrm{F}_{1}$ \\
\hline $\mathrm{F}_{2}$ & $\mathrm{~F}_{2}=0,5144$ & - & $\mathrm{F}_{2}$ & - & - & $\mathrm{F}_{2}$ \\
\hline$F_{3}$ & $F_{3}=0,5002$ & - & - & $\mathrm{F}_{3}$ & - & $\mathrm{F}_{3}$ \\
\hline $\mathrm{F}_{4}$ & $\mathrm{~F}_{4}=0,5004$ & - & - & - & $\mathrm{F}_{4}$ & $\mathrm{~F}_{4}$ \\
\hline
\end{tabular}

Figure 4 illustrates graphically the minimum values to the slope of the component degradation linear law for all four single faults considered, according to different process operational conditions. Such operational conditions of the process corresponding to the process controlled variables taking a minimum value of $10 \%$ and a maximum of $90 \%$.

Faults $F_{1}$ and $F_{2}$ are simulated through the change in the flow $D$. In this case, $\mathrm{F}_{1}$ could be simulated with a lower component degradation speed when the level is the maximum while $F_{2}$ could be simulated with a lower component degradation speed when the level is taking the minimum value. Faults $F_{3}$ and $\mathrm{F}_{4}$ have been simulated through the change in the flow $W$.

\begin{tabular}{|c|c|c|}
\hline Incipient faults & Minimal slope & Diagnosis time $[\mathrm{s}]$ \\
\hline $\mathrm{F}_{1}$ & 9.8 & 8.57 \\
\hline $\mathrm{F}_{2}$ & -90.2 & 0.94 \\
\hline $\mathrm{F}_{3}$ & 4.2 & 18.15 \\
\hline $\mathrm{F}_{4}$ & -39.5 & 2.01 \\
\hline
\end{tabular}

Table 9: Minimal slopes and detection time for single incipient faults $\left(M_{D}=M_{B}=90 \%\right.$ of $M_{D}$ and $M_{B}$ maximum).

degradation speed corresponds to faults $\mathrm{F}_{2}$ and $\mathrm{F}_{4}$. This is due to the fact that such faults corresponding to the blockage of the control valves fully close (flow tends to zero following a linear law with the slope presented in Table 7). As a consequence of that will occur an increase in the surge drum and reboiler levels, respectively, and so the fault isolation system has more time to isolate the faults and more incipient the faults can be. It is worth note that for all fault situations considered so far $100 \%$ of correct diagnosis were obtained.

From Tables 8 and 9 can be seen that similar results have been achieved with the setpoints of the process controlled variables taking the value $90 \%$. However, as far as the component degradation speed is concerned now an opposite situation is observed. From Table 9 can be seen that now the faults that can be simulated with a lower component degradation speed are $F_{1}$ and $F_{3}$ corresponding to the control valves blockage fully open. These results demonstrate that the performance of the fault isolation system under incipient fault scenarios depends on the process operational conditions.
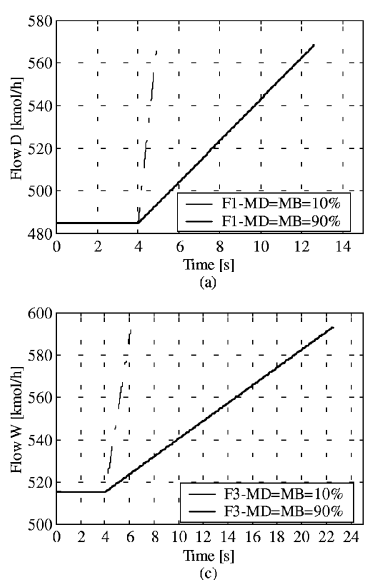

(c)
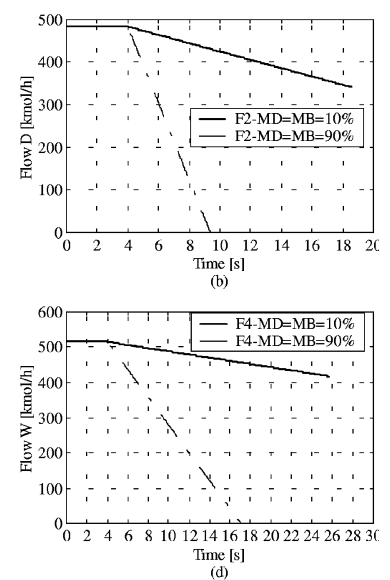

(d)

Figure 4: Minimal slopes of the single incipient faults $\left(F_{1}, F_{2}\right.$, $\mathrm{F}_{3}$ and $\mathrm{F}_{4}$ ).

In summary, under incipient fault scenarios the performance of the fault isolation system depends on the process operational conditions given by the values taken by the controlled variables $\left(M_{D}\right.$ and $M_{B}$ levels). The slopes achieved to the component degradation linear laws (see Figure 4) corresponding to the minimum component degradation speed that the fault isolation system is still able to isolate the correct fault. The minimum slopes to the component degradation linear law, allowing that the fault isolation system will isolate the correct fault, will be between the two traced lines in Figure 4 (for each fault) for all other possible process operational conditions. Of course, such operational conditions corresponding to all possible combinations of the values taken by the controlled variables.

Similar tests have been performed under double simultaneous incipient faults. Tables 10 and 11 show the results achieved. As a starting point the minimum slopes achieved to the component degradation linear law under incipient fault scenarios, have been used. However, it can be observed from Table 11 that with such values the HFNN only is able to isolate the correct faults under the double simultaneous fault situation, $\mathrm{F}_{2} \mathrm{~F}_{4}$. This is due to the fact that the performance of the fault isolation system is affected by the process operational conditions, as previously mentioned. For the other double simultaneous fault situations the slope of the components degradation linear laws have been changed until the HFNN isolate the correct faults.

Furthermore, as can be observed from the results presented in Table 11, the double simultaneous faults $\mathrm{F}_{1} \mathrm{~F}_{3}$ (valves blocked fully open) only are correct isolated if simulated with a

\begin{tabular}{|c|c|c|c|c|c|c|}
\hline \multirow{2}{*}{ Faults } & $\begin{array}{c}\text { Lower } \\
\text { Level }\end{array}$ & \multicolumn{4}{|c|}{ Medium Level } & $\begin{array}{c}\text { Upper } \\
\text { Level }\end{array}$ \\
\cline { 2 - 7 } & $\mathrm{FNN}_{0}$ & $\mathrm{FNN}_{1}$ & $\mathrm{FNN}_{2}$ & $\mathrm{FNN}_{3}$ & $\mathrm{FNN}_{4}$ & $\begin{array}{c}\text { OR } \\
\text { Operation }\end{array}$ \\
\hline $\mathrm{F}_{1} \mathrm{~F}_{3}$ & $\begin{array}{c}\mathrm{F}_{1}=0,8585 \\
\mathrm{~F}_{3}=0,9225\end{array}$ & $\mathrm{~F}_{1} \mathrm{~F}_{3}$ & - & $\mathrm{F}_{1} \mathrm{~F}_{3}$ & - & $\mathrm{F}_{1} \mathrm{~F}_{3}$ \\
\hline $\mathrm{F}_{1} \mathrm{~F}_{4}$ & $\begin{array}{c}\mathrm{F}_{1}=0 \\
\mathrm{~F}_{4}=0,5246\end{array}$ & - & - & - & $\mathrm{F}_{1} \mathrm{~F}_{4}$ & $\mathrm{~F}_{1} \mathrm{~F}_{4}$ \\
\hline $\mathrm{F}_{2} \mathrm{~F}_{3}$ & $\begin{array}{c}\mathrm{F}_{2}=0,5834 \\
\mathrm{~F}_{3}=0,0399\end{array}$ & - & $\mathrm{F}_{2} \mathrm{~F}_{3}$ & - & - & $\mathrm{F}_{2} \mathrm{~F}_{3}$ \\
\hline $\mathrm{F}_{2} \mathrm{~F}_{4}$ & $\begin{array}{c}\mathrm{F}_{2}=0,7551 \\
\mathrm{~F}_{4}=0,5468\end{array}$ & - & $\mathrm{F}_{2}$ & - & $\mathrm{F}_{4 \mathrm{v}}$ & $\mathrm{F}_{2} \mathrm{~F}_{4}$ \\
\hline
\end{tabular}

Table 10: Diagnosis results of double incipient faults ( $M_{D}=M_{B}=10 \%$ of $M_{D}$ and $M_{B}$ maximum). 


\begin{tabular}{|c|c|c|}
\hline Incipient faults & Minimal slope & Detection time $[\mathrm{s}]$ \\
\hline $\mathrm{F}_{1} \mathrm{~F}_{3}$ & $\mathrm{~F}_{1}=7000$ & $\mathrm{~F}_{1}=0,03$ \\
\hline $\mathrm{F}_{1} \mathrm{~F}_{4}$ & $\mathrm{~F}_{1}=5000$ & $\mathrm{~F}_{3}=0,01$ \\
\hline $\mathrm{F}_{2} \mathrm{~F}_{3}$ & $\mathrm{~F}_{4}=-630$ & $\mathrm{~F}_{4}=0,2$ \\
\hline $\mathrm{F}_{2} \mathrm{~F}_{4}$ & $\mathrm{~F}_{3}=-43$ & $\mathrm{~F}_{2}=1,83$ \\
\hline & $\mathrm{F}_{2}=-9,8$ & $\mathrm{~F}_{2}=7,86$ \\
\hline
\end{tabular}

Table 11: Minimal slopes and detection time for double incipient faults $\left(M_{D}=M_{B}=10 \%\right.$ of $M_{D}$ and $M_{B}$ maximum).

behaviour very close to the abrupt fault behaviour. This is due to the fact that under the current test conditions it has been assumed a value of $10 \%$ to the controlled variables corresponding to the minimum values of $\mathrm{M}_{\mathrm{D}}$ and $\mathrm{M}_{\mathrm{B}}$ levels. Therefore, under that operational conditions the double simultaneous faults, $\mathrm{F}_{1} \mathrm{~F}_{3}$, must be simulated with a higher degradation component speed in order to be isolated before the levels reach the value zero. When simulating faults that produce an opposite effect (valves blocked fully closed), it can be observed an improvement in the performance of the fault isolation system. This is because the faults effect makes an increase in the levels values allowing more time to the HFNN structure to isolate the correct faults.

Thus, Table 11 presents the minimum values achieved to the slope of the components degradation linear law providing $100 \%$ of correct diagnosis under double incipient fault scenarios (see Table 10). It is worth note, that when the setpoint of the controlled variables are taking the value $90 \%$ corresponding to the levels maximum value, it has been observed an opposite behaviour to the fault isolation system. Now, in order to achieve the correct diagnosis the double simultaneous incipient faults, $\mathrm{F}_{2} \mathrm{~F}_{4}$, need to be simulated with a higher component degradation speed while $F_{1} F_{3}$ can be simulated with a more incipient behaviour.

\section{Conclusions}

An on-line fault isolation system for industrial actuators, consisting on a HFNN structure, has been implemented. The fault diagnosis system based on a FNNs hierarchical structure combines the advantages of both, fuzzy reasoning and neural networks. Successful results have been achieved during simulation studies conducted with a CBDC plant.

It has been demonstrate that the current fault isolation approach is able to diagnose multiple simultaneous abrupt and incipient faults from only single fault symptoms. During the current study, it has been observed that the neural network's generalization ability has a great importance in the diagnosis of incipient faults since the training patterns only include single abrupt fault symptoms.

Since in the current approach the relationships between faults and faults symptoms are distributed through several FNNs, one can conclude that the HFNN training can be done more easily than a non-hierarchical neural network system for the same purpose.
The successful results achieved during the simulation studies conducted on the CBDC process let predict great potential for the use of the architecture proposed in this paper with real plants.

\section{Acknowledgement}

This research was partially supported by the EU FP V Research Training Network DAMADICS and program POCTI, FCT, Ministério da Ciência e da Tecnologia, Portugal.

\section{References}

[1] J. M. F. Calado, J. M. G. Sá da Costa. "An Expert System Coupled with a Hierarchical Structure of Fuzzy Neural Networks for Fault Diagnosis", International Journal of Applied Mathematics and Computer Sciences, volume 9, n. 3, pp. 667-687, (1999).

[2] J. M. F. Calado, J. M. G. Sá da Costa. "A Fault Detection and Diagnosis Methodology for Chemical Processes", 14th IFAC Word Congress, volume O, pp. 539-544, (1999).

[3] J. Chen. "Robust Residual Generation for ModelBased Fault Diagnosis of Dynamic Systems", Ph.D. Thesis, Dept. of Electronics, University of York, UK, (1995).

[4] J. Ingham, I. J. Dunn, E. Heinzle, J. E. Prenosil "Chemical Engineering Dynamics - Modelling with PC Simulation", VCH, (1994).

[5] S. Haykin. "Neural Networks - A Comprehensive Foundation", Macmillan College Publishing Company, Inc., (1994).

[6] J. Korbicz. "Neural networks and their application in fault detection and diagnosis", Proc. IFAC Symp. on Fault Detection, Supervision and Safety for Technical Processes SAFEPROCESS'97, volume 1, pp. 377-382, (1997).

[7] J. Korbicz, J. Kus. "Diagnosis of Sugar Factory Processes using GMDH Neural Networks", 4th IFAC Symp. on Fault Detection, Supervision and Safety for Technical Processes SAFEPROCESS'2000, volume 1, pp. 343-347, (2000).

[8] D. Nauck, F. Klawonn, R. Kruse. "Foundations of Neuro-Fuzzy Systems", John Wiley \& Sons, (1997).

[9] R. J. Patton, J. Chen, T. M. Siew. "Fault Diagnosis in Non-linear Dynamic Systems via Neural Networks", Proc. IEEE Int. Conference CONTROL'94, volume 2, pp. 1346-1351, (1994).

[10] M. Riedmiller, H. Braun. "A direct adaptive method for faster backpropagation learning: The RPROP algorithm", In Proc. of the IEEE Intl. Conf. on Neural Networks, pp. 586-591, (1993).

[11] K. Watanabe, S. Hirota, L. Hou, D. M. Himmelblau. "Diagnosis of Multiple Simultaneous Fault via Hierarchical Artificial Neural Networks", AIChE Journal, volume 40, n. ${ }^{\circ}$ 5, pp. 839-848, (1994). 